Canadian University Music Review

Canadian University Music Review

Revue de musique des universités canadiennes

\title{
L'Énigme de la première édition (1624) des Capricci de Girolamo Frescobaldi
}

\section{Étienne Darbellay}

Numéro 3, 1982

URI : https://id.erudit.org/iderudit/1013831ar

DOI : https://doi.org/10.7202/1013831ar

Aller au sommaire du numéro

\section{Éditeur(s)}

Canadian University Music Society / Société de musique des universités canadiennes

\section{ISSN}

0710-0353 (imprimé)

2291-2436 (numérique)

Découvrir la revue

Citer cet article

Darbellay, É. (1982). L’Énigme de la première édition (1624) des Capricci de Girolamo Frescobaldi. Canadian University Music Review / Revue de musique des universités canadiennes, (3), 123-157. https://doi.org/10.7202/1013831ar

All Rights Reserved ( C Canadian University Music Society / Société de musique des universités canadiennes, 1982
Ce document est protégé par la loi sur le droit d'auteur. L'utilisation des services d'Érudit (y compris la reproduction) est assujettie à sa politique d'utilisation que vous pouvez consulter en ligne.

https://apropos.erudit.org/fr/usagers/politique-dutilisation/ 


\title{
L'ÉNIGME DE LA PREMIÈRE ÉDITION (1624) DES CAPRICCI DE GIROLAMO FRESCOBALDI ${ }^{1}$
}

\author{
Étienne Darbellay
}

La nature iconoclaste de la contribution qui va suivre ne manquera pas de heurter les sentiments " holistiques » de ceux qui, parmi les musicologues, cherchent à tout prix à retrouver, dans un opus homogène du XVIIe siècle, ces fils conducteurs qui, à l'instar des cycles organiques du XIXe siècle, pourraient ésotériquement réunir dans une perspective globale un ensemble de compositions regroupées le plus souvent selon des critères fonctionnels ou simplement génériques.

Bien malgré nous en effet, il a fallu nous rendre à l'évidence : non seulement l'ordre des Capricci frescobaldiens de 1624, mais encore leur nombre et même, pour trois d'entre eux, leur organicité compositionnelle interne résultent vraisemblablement dans leur forme définitive d'aléas extérieurs plutôt que de l'intention originelle du compositeur.

Le 12 avril 1624, Frescobaldi dédie ce qui constitue à n'en pas douter son chef d'oeuvre dans le domaine de la variation contrapuntique - les douze Capricci pour clavier - au prince de Modène Alfonso d'Este. Il renoue par là d'anciens liens avec sa patrie d'origine, Ferrare, et, à travers la personnalité révérée de son maître Luzzasco Luzzaschi expressément nommé dans la dédicace, rend hommage à "l'antique et héréditaire protection des beaux-arts " dont les d'Este, qui l'employèrent, pouvaient s'enorgueillir.

Le volume, issu en 1624 des presses de l'imprimeur romain Luca Antonio Soldi dont c'est l'un des derniers imprimés connus, ${ }^{2}$ est typographié sans beauté mais proprement. Deux étapes de

Revue de musique des universités canadiennes, $\mathrm{N}^{\circ} 3,1982$. 
corrections au moins furent entreprises. Dans un premier temps, nombre d'altérations furent introduites hors portée sur les plaques elles-mêmes sans qu'il soit possible de déterminer s'il s'agit d'améliorations d'auteur ou de corrections d'imprimerie : rien ne distingue les altérations incorporées à la portée de celles qui sont ajoutées hors portée; dans les deux cas on rencontre aussi bien des altérations "nécessaires " - ou qu'un exécutant avisé aurait automatiquement introduites - que des altérations arbitraires résultant évidemment du choix délibéré du compositeur. Les exemplaires terminés ont en outre été revus dans une seconde étape, comme en témoignent les nombreuses corrections manuscrites à l'encre - quasi identiques sur la majorité des copies conservées - et réalisées souvent par la même main, donc chez l'imprimeur lui-même. A de rares exceptions près, c'est la leçon ainsi amendée qui fut reprise dans les éditions ultérieures de Vincenti, ${ }^{3}$ où les altérations hors portée de 1624 sont incorporées à la portée et les corrections manuscrites tenues pour version authentique. Comme les éditions vénitiennes introduisent toutefois de nouvelles erreurs, c'est en définitive l'édition originale de Soldi qui constitue la source la plus fiable des Capricci.

\section{Foliotage et pagination}

Si l'on tient compte du soin relativement scrupuleux avec lequel fut parachevée la typographie musicale du volume, on se trouve alors d'autant plus surpris par l'incroyable négligence dont atteste sa mise en ordre définitive : la moitié dela pagination manque ; celle qui existe présente plusieurs incohérences ; le foliotage apparaît parfaitement fantaisiste, et la Tavola (table du contenu) inverse l'ordre de deux pièces et donne une pagination qui est non seulement absente du volume mais ne correspond pas à celle qu'on peut reconstituer. Attribuer ces incohérences à la seule incompétence de Soldi ne cadre ni avec l'expérience qu'il avait nécessairement acquise après cinq ans au moins dans le métier ni avec le soin de la partie musicale du travail. Elles constituent évidemment les séquelles d'un bouleversement qui a dû intervenir en cours d'élaboration de l'ouvrage, et dont on peut d'ores et déjà tenir Frescobaldi pour responsable, cela pour deux raisons : 1) nous avons pu prouver ailleurs qu'il sera responsable en 1637 d'un bouleversement analogue dans le contexte de l'édition définitive des deux livres de Toccate $;^{4}$ le cas des Capricci en constituerait dès lors un simple précédent. 2) Il est impensable qu'un imprimeur en possession d'un manuscrit définitif et bien 
organisé dont il réalise la typographie dans l'ordre, parvienne à folioter ses plaques avec une telle inconséquence, tant dans la constitution des cahiers que dans leurs identifications internes.

Avant de présenter ces inconséquences, nous pensons simplifier la tâche du lecteur en lui rappelant la démarche normale du foliotage. Un folio, constitué d'une feuille oblongue pliée en deux, comporte quatre pages. Un cahier est formé de plusieurs folios, dont le nombre peut augmenter de deux à huit en moyenne lorsque diminue le poids du papier employé. Soldi use d'un papier lourd et ne dépasse pas trois folios par cahier. ${ }^{5}$ Normalement l'identification du cahier se fait par une let tre et celle du folio par un chiffre : A(1), A2, A3 par exemple constituent un cahier de douze pages, dont le folio $A(1)$ portera la pagination 1-2-11-12, A2 3-4-9-10, et A3 5-6-7-8 ; les foliotages A(1)-3 figureront évidemment au pied des pages impaires 1,3 et 5 respectivement, aucune des autres n'étant identifiée par autre chose que sa pagination.

Il est dès lors évident qu'un imprimeur devrait logiquement paginer son ouvrage en cours de typographie puis le folioter une fois terminé en fonction du nombre de pages et des problèmes de reliure. Il est aussi envisageable qu'il procède à l'inverse, réalisant la pagination après le foliotage : le risque d'erreur est cependant plus grand, et le foliotage réalisé parallèlement à la typographie suppose qu'il connaisse à l'avance le nombre exact de pages qu'il remplira, au moins pour chaque cahier. (La typographie permettait en effet, grâce aux caractères plus ou moins grands de portée vide disponibles, une certaine marge de manoeuvre à même de compenser les erreurs d'évaluation dans l'analyse du mansucrit.) Comme nous le verrons, c'est cette seconde solution qui fut choisie par Soldi.

Nous avons examiné sur place presque tous les exemplaires conservés de cette édition à l'exception de ceux de Rome, Berlin et Washington qui ont été compilés sur la base de microfilms et de xérocopies. ${ }^{6}$ Tous sont reliés de la même façon et sont identiques quant aux indices pertinents au présent examen. ${ }^{7}$ La complexité de la situation nous oblige à l'exposer sous la forme d'un tableau du foliotage actuel, qui décrit l'organisation des cahiers dans le volume : les quatre premiers cahiers de trois folios (pp. 1-12, 13-24, 25-36 et 37-48) sont suivis d'un cahier de deux folios (49-56), d'un cahier de trois folios ([57] - [68]), de trois cahiers de deux folios sans pagination ([69-76, 77-84, 85-90]) et d'un folio final ([93-96]) (voir Tableau I ci-contre). 


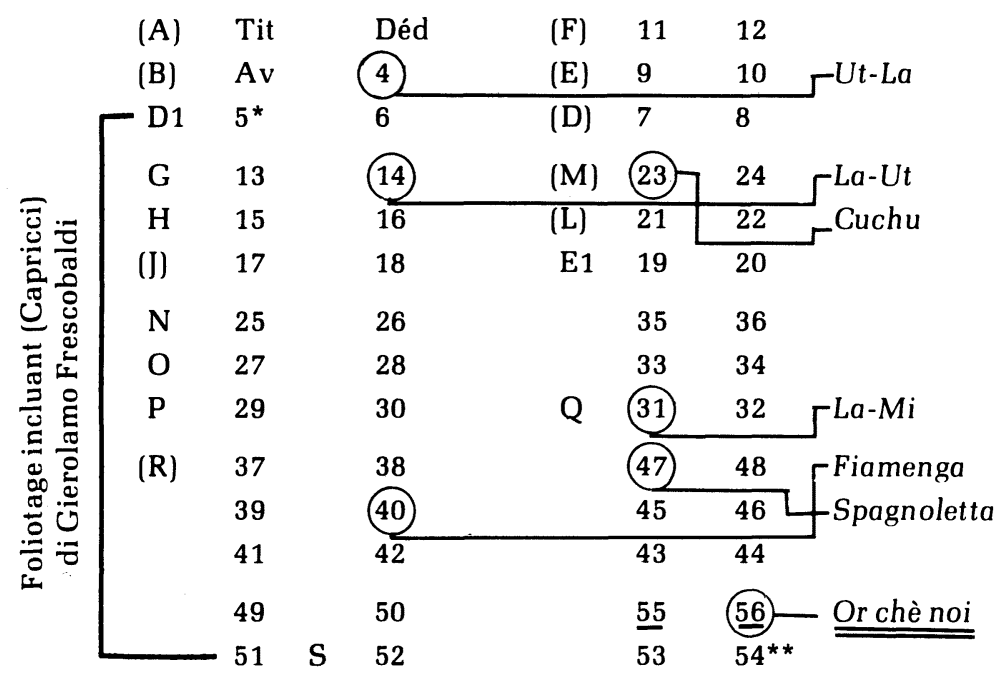

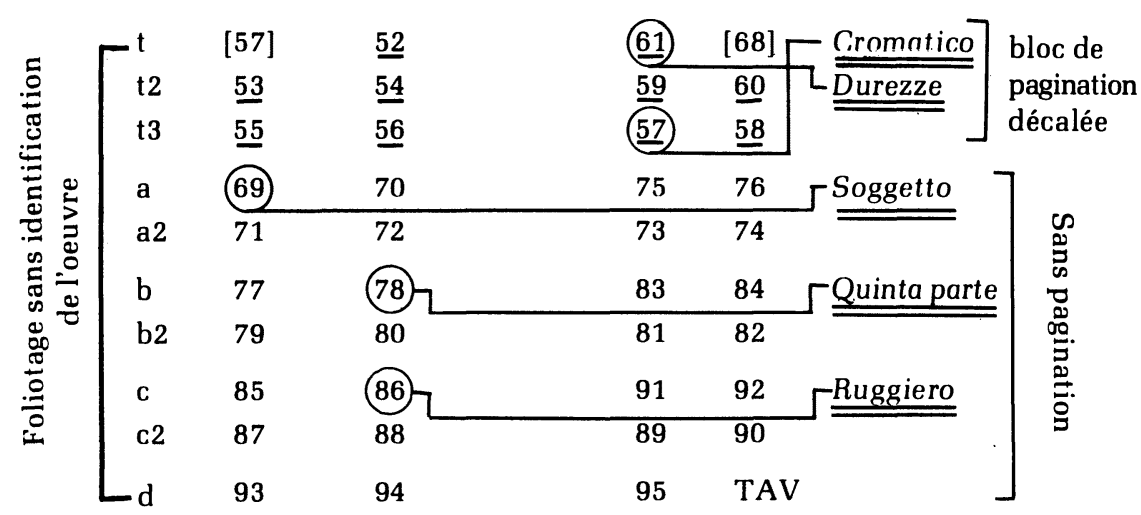

Tableau I

Le foliotage actuel

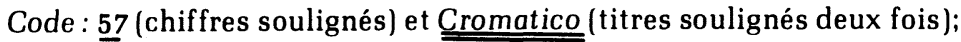
petits caractères dans l'original.

${ }^{*}=$ chiffre à droite de la page ;

** $=$ chiffre à gauche de la page. 
Frappante est d'emblée cette disparité des cahiers: pourquoi Soldi n'a-t-il pas simplement composé son volume de huit cahiers de trois folios par exemple (quatre-vingt-seize pages) ou de douze cahiers de deux folios? Le titre de la pièce en cours est répété au sommet de chaque page du volume (avec plusieurs erreurs et différences d'un exemplaire à l'autre d'ailleurs) : on note que ceux des six premiers Capricci sont typographiés en gros caractères et ceux des six derniers, en petits caractères (soulignés deux fois dans notre tableau). Il en est de même pour les chiffres de pagination existants, les petits chiffres étant soulignés dans notre tableau. A cet égard, le sixième cahier présente une anomalie: les numéros de page 57 et 68 manquent, et le reste du bloc est paginé 52 à 61 , avec par conséquent un décalage de six pages par rapport au décompte normal; dès le cahier suivant, la pagination manque jusqu'à la fin du volume. Les chiffres pairs enfin figurent toujours à gauche de la page et vice-versa : cette disposition est inversée pour les pages 5 et 54 .

Le plus surprenant demeure sans doute le foliotage qui nous dicte une remarque importante : toute se passe comme si, dans la première partie du volume (pp. 1-56), la rectitude dela pagination devait suppléer l'incohérence apparente du foliotage alors que l'inverse est vrai de la seconde partie, où un foliotage relativement intelligible permet d'organiser le volume en dépit des déficiences ou de l'absence de pagination. On remarqera en outre que le foliotage lui-même procède de trois générations distinctes : à la première appartiennent les lettres $\mathrm{D} 1, \mathrm{E} 1$ et $\mathrm{S}$ (p. 52) qui ne sont pas à leur place ; à la seconde, toutes les autres lettres majuscules qui ne constituent pas un foliotage normal mais une "identification par paires » de pages où, à l'exception de $Q$, seules sont indiquées les trois paires initiales de chaque cahier. On peut en effet suivre la série de $A$ à $Q$ en suppléant les lettres manquantes (entre parenthèses dans notre tableau) $A-F$ pour le premier cahier, $\mathrm{J}-\mathrm{M}$ pour le deuxième et éventuellement $\mathrm{R}-\mathrm{S}$ pour le troisième. (On peut aussi s'arrêter à $Q$, supposer $R$ pour le quatrième cahier et tenir le $\mathrm{S}$ de la page 52 , demeuré de la génération antérieure, comme suffisant pour identifier le cinquième cahier avant le sixième qui est noté $t$ ). Ces deux premières générations ont en commun des lettres majuscules et la présence de l" " adresse " (Capricci) di Gierolamo Frescobaldi. La troisième génération est représentée par les lettres minuscules, sans " adresse " de l'oeuvre, mais constituant un véritable foliotage avec lettres et chiffres. La seule question qui peut être soulevée à ce stade est 
celle de savoir pourquoi t 1-3 est suivi de a à d et non pas de uà $\mathrm{z}$ : il y a manifestement rupture entre ces deux "blocs ", rupture corroborée d'ailleurs par le problème de la pagination.

Explicitons maintenant le premier raisonnement sousjacent à notre classification des " générations ". Il est a priori évident que la troisième génération fut la dernière introduite : le processus normal est en effet de folioter d'abord en lettres majuscules puis, par exemple, en doubles lettres ; l'usage des minuscules n'a d'autre but que de distinguer l'identification de ces derniers cahiers non paginés de celle des cahiers initiaux, par conséquent préexistante. D'autre part l'absence de l'adresse, normalement présente à cette époque, indique aussi que par ce foliotage, on a "paré au plus urgent ", alors que dans le foliotage antérieur évidemment bouleversé, on avait pris la peine de la noter. Le bloc final, dès " a " (page [69]), constitue donc soit un ajout en fin de course (non prévu dans un foliotage original) soit un « transfuge " d'une autre section du volume. Du bloc « $t$ », on peut affirmer qu'il a dû, dans une étape antérieure, occuper réellement la place que lui at tribue sa pagination devenue erronée - dans la version actuelle : le Capriccio Or chè noi rimena devait débuter à la page 50 et non 56 . (Nous verrons plus loin que ce saut de six pages, qu'on pourrait a priori considérer comme une simple erreur, trouvera sa justification.)

Le problème de la chronologie des deux premières générations est simple : puisque les lettres D1, E1 et S n'ont de toute évidence aucun rapport avec l'ordre actuel du volume, elles constituent nécessairement les témoins, demeurés (heureusement) là par inadvertance, d'un état antérieur à celui que représentent les autres lettres. L'inconséquence de ces dernières notamment dans l'absence de $C$, J et la présence de $Q$ - atteste d'autre part l'existence d'un stade intermédiaire où ces lettres occupaient adéquatement les positions d'un foliotage cohérent: $Q$ par exemple provient vraisemblablement d'un foliotage différent, avec adresse de l'oeuvre, et situé dans la colonne de gauche du tableau.

Afin de parachever l'énoncé des données, il convient maintenant d'examiner la TAVOLA que nous reproduisons ci-après, en fac-similé. 

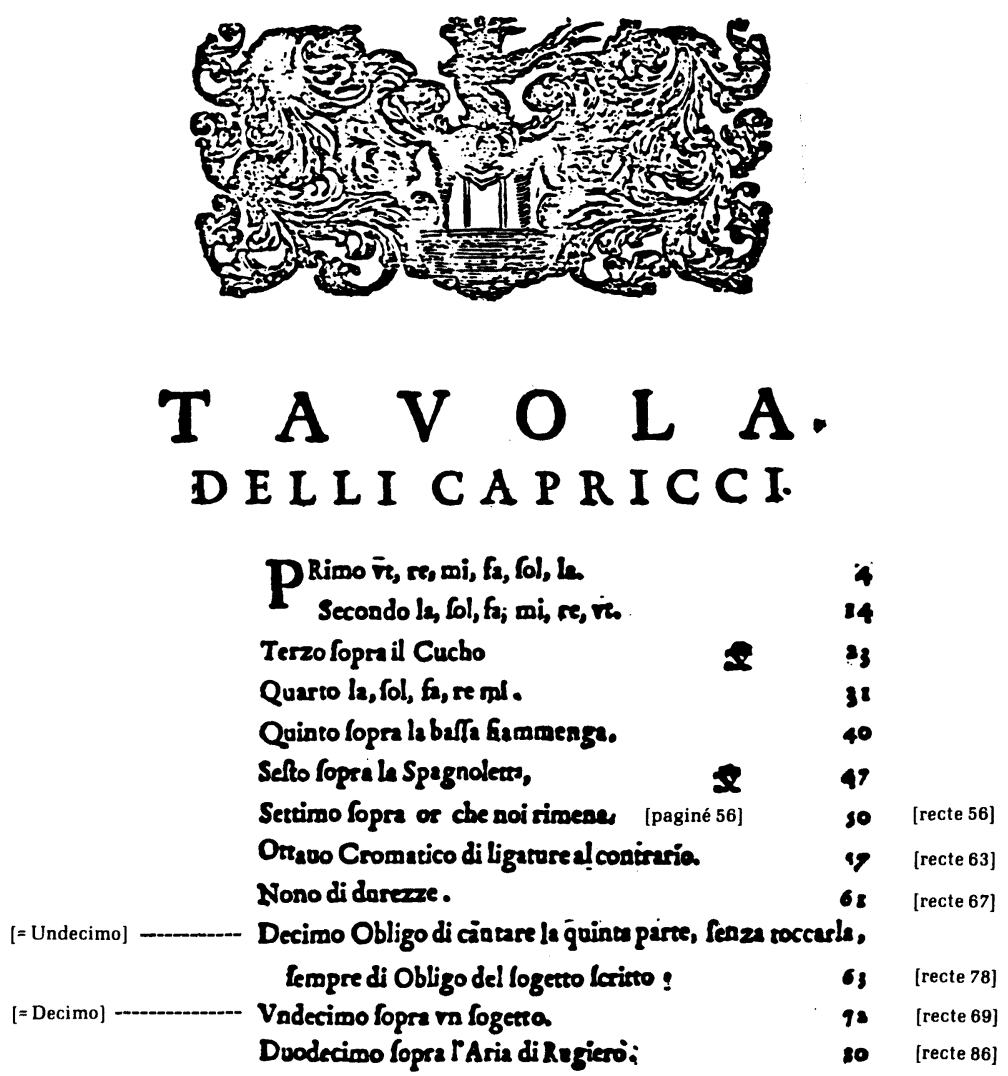

I L E IN B. 
Les erreurs mêmes de cette table - que nous corrigeons en marge - constituent de nouveaux indices. S'il est fréquent de rencontrer dans une table des erreurs occasionnelles de pagination, il est impossible dans le cas présent d'attribuer le décalage de six pages dans la pagination du bloc final, dès le Capriccio VII, à l'étourderie du typographe: de telles erreurs reproduisent normalement des erreurs de pagination dans le volume. Or la pagination, nous l'avons vu, manque dès la page 62; le typographe l'a donc déduite en connaissant le nombre de pages de chaque pièce pour les derniers Capricci. Là encore, l'inversion d'ordre des Capricci X et XI est surprenante, d'autant plus que la pagination reconstituée (63 et 72) est celle qui correspond à l'ordre des pièces dans le volume, tenu compte du décalage de six pages dès la page 50. En revanche tout est correct dans le premier bloc, jusqu'à la page 47 incluse. Cette table trahit donc les séquelles d'un état intermédiaire du volume, où le Capriccio VII débutait réellement à la page 50 (telle quelle dans la table mais corrigée en 56 dans le volume); d'un état ultérieur où les six pages manquantes du début ont été ajoutées sans pour autant que la pagination du bloc central ait été déjà " corrigée »; et vraisemblablement d'un état où l'ordre des Capricci sopra un Sogetto et Obligo di cantare la quinta parte était inversé.

L'ensemble de ces déductions nous est permis dans la mesure où les erreurs de la table ne font que confirmer le bouleversement du volume : si l'on isolait en effet les données observables dans la table de celles que nous connaisons déjà, rien ne nous autoriserait à les tenir pour autre chose que de simples étourderies. C'est à partir du moment où nous avons de sérieuses raisons de penser que ce désordre peut être imputé à un processus de modification plutôt qu'à de pures distractions que nous sommes en droit d'en considérer les détails particuliers comme des indices utilisables.

Jusqu'à ce point, nous nous sommes indiscutablement mû sur un terrain ferme ; l'ensemble des données irréfutables présentées étayent le constat suivant : bien loin d'attester l'incompétence d'un imprimeur débutant, le produit final que nous avons sous les yeux résulte clairement d'une réorganisation hâtive et inachevée d'un ensemble dont non seulement l'ordre, mais vraisemblablement aussi le contenu a dû faire l'objet de modifications imprévues. De plus, comme trois étapes au moins peuvent être d'emblée 
mises à jour dans le processus, il appert que la charge doive enêtre certainement imputée au compositeur plutôt qu'à l'imprimeur:en supposant que l'imprimeur ait disposé d'un manuscrit parfait et complet au départ, il serait pratiquement inconcevable qu'il ait dû, à la suite d'erreurs multipliées, s'y reprendre à trois fois au moins pour venir à bout de son foliotage. Il est en revanche possible, comme nous le verrons, que certains amendements introduits par Frescobaldi l'aient été à la suite de modifications rendues nécessaires par une erreur ou une omission du typographe.

Nous allons donc tenter maintenant de reconstituer l'ensemble du processus en adoptant pour règle de conduite celle du «plus court chemin ", c'est à dire en nous contentant toujours de l'hypothèse qui offre l'explication la plus simple des indices relevés. Il va de soi que la réalité a pu être plus complexe que le reflet que nous tenterons d'en donner : si nous avons le choix entre deux ensembles d'indices conduisant à des scénarios différents mais aboutissant aux mêmes résultats finaux, nous choisirons celui qui se base sur les indices les plus probants, sans nier toutefois la plausibilité de l'autre.

\section{Le projet originel}

Le point de départ évident est celui des lettres de foliotage D1-E1, aux pages 5 et 19. Ces lettres, qui se suivent, sont séparées par quatorze pages, et doivent donc nécessairement provenir d'un foliotage de cahiers et non d'un foliotage par "paires», comme d'ailleurs le confirment les chiffres. Le problème est cependant le nombre de pages impliquées : un cahier est constitué de huit, douze ou seize pages, si bien que nous avons le choix entre retrancher deux ou six pages ou en ajouter à la rigueur deux autres. La dernière solution doit être écartée d'emblée: elle amènerait à supposer a) des cahiers de quatre folios, que Soldi a clairement évités, et b) que Soldi (ou Frescobaldi) aurait sacrifié deux pages déjà réalisées, ce qui représente l'hypothèse la moins vraisemblable pour de multiples raisons. ${ }^{8}$ Demeurant fidèle au principe énoncé plus haut, nous devons donc chercher la solution qui cadre le mieux avec le donné existant et entraîne le moins de bouleversements possible. Retrancher deux pages seulement s'avère impossible sans toucher au texte musical dont la continuité est évidente. En examinant de près le Capriccio I cependant, on constatera non sans surprise que la quatrième page (page 7) se termine avec une double barre, à la fin d'une variation (mesure 76) : or à trois autres exceptions près (qui toutes trouveront une expli- 
cation analogue à celle qui va suivre), jamais une variation n'est close par une double barre avant la fin du morceau. On peut donc admettre que le Capriccio aurait pu se terminer là dans une étape antérieure - soit par la volonté de Frescobaldi, soit moins vraisemblablement à la suite d'un " court circuit » dans le manuscrit ou la typographie. ${ }^{9}$ On pourrait dès lors retrancher sans autre modification les pages 8 à 13, qui représentent précisément les six pages en excès, ce qui nous donnerait le foliotage suivant :

$\begin{array}{cllll}\text { D1 } & 5 & 6 & 17 & 18 \\ \text { (D2) } & 7 & (14) & 15 & 16 \\ \text { E1 } & 19 & 20 & & \\ (\mathrm{E} 2) & 21 & 22 & \ldots . & \end{array}$

Notons au passage - nous y reviendrons plus loin - que l'hypothèse est d'autant plus vraisemblable qu'elle semble être confirmée par le " trou " de six pages précisément qui perturbe la pagination du bloc « $t$ ».

$\mathrm{D} 1$ et $\mathrm{E} 1$ forment maintenant le quatrième et cinquième cahiers du volume : le problème suivant est par conséquent de retrouver les trois premiers cahiers ( $\mathrm{A}$ à $\mathrm{C}$ ). Là également, l'évidence même est qu'ils se trouvent dans le volume actuel. Au cas contraire, il faudrait supposer que Frescobaldi aurait sacrifié vingt pages au moins déjà typographiées (trois cahiers de huit pages au minimum, déduction faite des trois pages initiales non numérotées avec titre, dédicace, avertissement et de la première page du Carpiccio Ut re mi fa sol la), ce qui est exclu pour les raisons déjà données - que ces vingt pages aient contenu d'autres Capricci ou quelqu'autre groupe de compositions connues ou inconnues.

Ce stade de l'investigation relève du véritable exercice sur la corde raide. Pour ne pas tomber dans l'arbitraire le plus absolu, il nous faut injecter de nouveaux éléments dans le circuit. Le plus simple est de nous occuper d'abord de la lettre de foliotage $\mathrm{S}$ qui figure à la page 52 (Spagnoletta). Il est évident que cette page devait être impaire puisqu'elle comprend un foliotage, et qu'elle devait se trouver au début d'un folio. Or de E1 à S, le chemin à parcourir est bien long si l'on suppose à chaque lettre un cahier de huit pages ; on arriverait à un total de cent pages à combler avant même $S$, ce qui est manifestement exclu. Nous noterons cependant l'absence de chiffre après $S$ d'une part, et d'autre part l'existence de l'identification par " paires " dans le début du foliotage actuel du volume : c'est évidemment à ce système que Soldi a dû 
recourir - pour les raisons qui seront exposées plus loin. Le nombre de pages à remplir, entre la fin de Capriccio II et la sixième page de la Spagnoletta (folio S) se trouvera ainsi réduit à 28 .

On pourrait objecter ici que le foliotage a bien pu se faire à raison d'une lettre par folio et non par paire de pages, ce qui amènerait ce total à cinquante-deux pages (douze fois quatre pages + quatre pages in E1); le volume aurait donc vingt-quatre pages avant $D 1$, plus soixante-quatre pages jusqu'à $S$ plus cinq pages au moins depuis $S$ (les quatre dernières de la Spagnoletta et la Tavola), ce qui ferait un total minimum de quatre-vingt-seize pages. Or nous devons, dans ce décompte, retrancher les six pages du Capriccio I du total actuel de quatre-vingt-seize, ce qui donne un maximum de quatre-vingt-dix pages. D'entrée cette solution est donc à écarter. Nous savons dès lors que la section qui suit E1 comprendra vingt-huit pages jusqu'à $S$ et que la section qui précède $\mathrm{D} 1$ devrait être composée de trois cahiers de douze pages chacun (A3 - C3): ${ }^{10}$ notre total avant $S$ est ainsi ramené a soixante-seize pages plus $S$ (minimum cinq pages), donc quatrevingt-un environ, ce qui est plausible.

Ainsi se fait jour peut-être la raison d'être du chiffre «l " qui suit les lettres $\mathrm{D}$ et $\mathrm{E}$ : après un premier groupe de trois cahiers de douze pages, vraisemblablement foliotés selon la tradition A A2 - A3 etc., donc sans l'inutile " $\mathrm{l}$ " après $\mathrm{A}$, Soldi a entamé des cahiers de deux folios qu'il a distingués des précédents par l'ajout du chiffre. Après E1, il s'engage dans l'identification par paires. La raison de ce procédé étrange apparaît simple à la réflexion, si l'on se souvient du raisonnement que nous avons fait plus haut: le typographe qui est en possession d'un manuscrit définitif peut sans problème planifier ses cahiers aussitôt qu'il connaît le nombre de pages typographiées. La planification initiale par trois montre qu'il était bien en possession d'un ensemble « définitif » en tout cas jusqu'à la page 35 , réservant peut-être la page 36 pour une tavola. C'est alors que Frescobaldi, devant la minceure relative du volume (cet argument sera étayé plus bas), lui amène les Capricci I et II (Ut re mi fa sol la et La sol fa mi re ut) dont sera formé l'essentiel des cahiers $\mathrm{D}$ et $\mathrm{E}$ - planifiés dès lors en système de deux folios. A ce point - peut-être à cause du trou de trois pages avant la tavola dans le cahier E - fut vraisemblablement poursuivi ce processus d'alimentation du volume pièce à pièce, ce qui expliquerait l'identification par paires comme système de substitution au foliotage : étant dès cet instant dans l'incertitude quant au nombre de pages encore à venir, et donc quant à l'organisation 
globale du volume en cahiers, le typographe recourt alors au marquage de chaque paire de plaques par une lettre, ce qui lui laisse toute liberté quant à leur ordre d'impression subséquent sur les folios et par conséquent quant à la reliure finale en cahiers. (Après deux lettres, il peut parfaitement en sauter deux autres en pensant à des cahiers de deux folios, sauf à rajouter ultérieurement les lettres absentes s'il se décidait pour des cahiers de trois folios par exemple.)

Nous avons maintenant sensiblement réduit l'horizon des possibilités : nous devons remplir vingt-huit pages de musique de façon à retrouver $\mathrm{S}$ comme page 77 du volume, dans la marge de gauche d'un tableau hypothétique. Cette page 77 est la sixième page de la Spagnoletta. En examinant la typographie, on constatera que la fin de la Bassa Fiamenga, qui dans l'ordre actuel précède la Spagnoletta, empiète d'une mesure sur la page initiale de cette dernière (page 47 actuelle; cf. le fac-similé) : c'est le seul cas dans le volume où le début d'un nouveau Capriccio ne correspond pas au début d'une nouvelle page. Il faut par conséquent conclure que ces deux Capricci se suivent nécessairement.

Une remarque doit être amenée à ce point : la première ligne de cette page 47 a) présente la plus grande concentration d'erreurs de tout le volume, et b) est typographiée de façon extrêmement serrée. Parmi les erreurs, on note particulièrement deux éléments : d'abord un décalage significatif d'une mesure vers la gauche, sous la portée, de trois liaisons consécutives au ténor, comme si le ténor avait été déplacé vers la droite alors que demeuraient en place les liaisons ; ensuite, dans le soprano de la mesure finale de la Bassa Fiamenga, le seul cas d'inversion de la haste d'un $\mathrm{si}_{3}$ au soprano par rapport à sa direction normale (vers le haut), cela sur tous les exemplaires sauf un: ${ }^{11}$ trop serré, le caractère a dû en effet sauter en cours d'impression et être replacé sens dessus dessous. Tout se passe donc comme si la Spagnoletta avait été typographiée d'abord, suivie de la Fiamenga et qu'en conséquence d'une erreur de calcul, le ty pographe qui ne parvenait pas à terminer le morceau sur la page, ait refait les deux premières lignes de la Spagnoletta - primitivement bien espacées comme c'est normalement le cas du début des autres Capricci - de façon à y introduire cette mesure finale. Ainsi se trouvait inversé l'ordre prévu Spagnolette-Fiamenga qui, en raison de l'animation d'écri- 
Capricio Soprala Spagnoletta

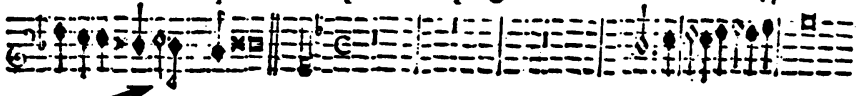
$\longrightarrow$ 落:

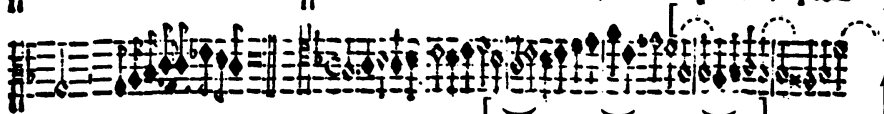

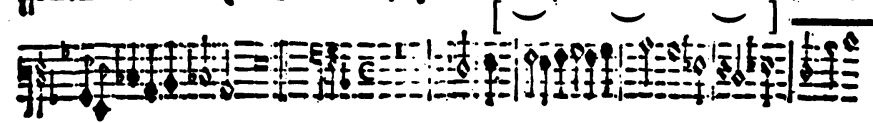

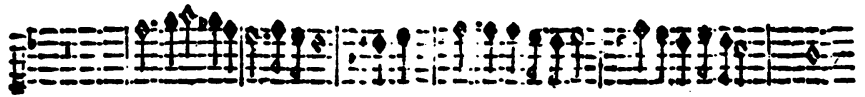

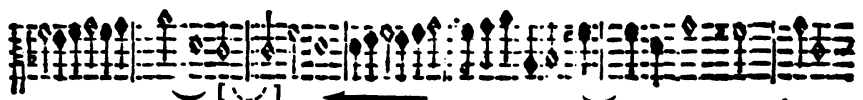

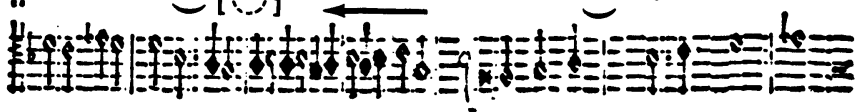

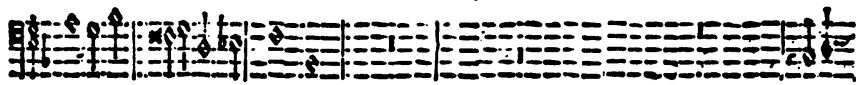

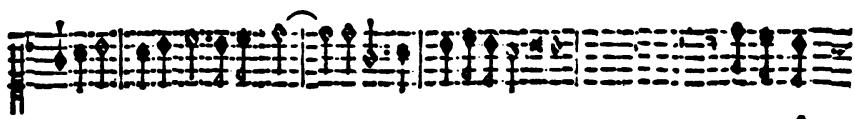

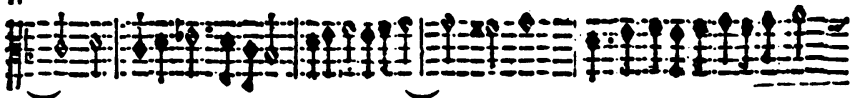

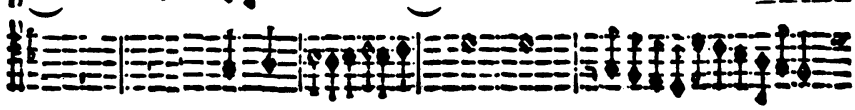

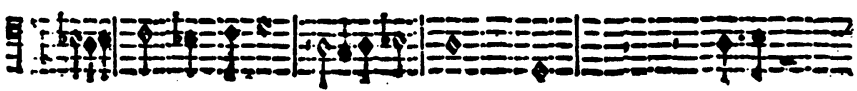


ture particulière de la Fiamenga, aurait été de fait infiniment préférable au cas où le volume se serait terminé là.

Si nous revenons maintenant à nos cahiers, nous obtenons la situation suivante :

\begin{tabular}{|c|c|c|c|c|c|}
\hline \multirow[t]{2}{*}{$\rightarrow \mathrm{E} 1$} & 45 & 46 & $x$ & $x$ & \multirow[t]{2}{*}{$\Gamma$ fin de La sol fa mi re ut } \\
\hline & 47 & 487 & $x$ & $x$ & \\
\hline$(\mathrm{F})$ & $x$ & $\mathbf{x}$ & $x$ & $x$ & \\
\hline (G) & $x$ & $x$ & $x$ & $x$ & \\
\hline$(\mathrm{K})$ & $x$ & $x$ & 67 & 68 & \\
\hline (L) & $x$ & $x$ & (40) & 66 & $\ulcorner$ Bassa Fiamenga \\
\hline (O) & 69 & 70 & 75 & 76 & \\
\hline (P) & 71 & (47) & 73 & 74 & $\ulcorner$ Spagnoletta \\
\hline$\rightarrow \mathrm{S}$ & 77 & 78 & $x$ & Tavola? & \\
\hline$(\mathrm{T})$ & 79 & 80 & $x$ & $x$ & \\
\hline
\end{tabular}

Une disposition en cahiers de trois folios serait également envisageable : toutefois elle est moins vraisemblable et, en outre, elle ne rendrait pas compte comme nous le verrons de l'irrégularité des identifications alphabétiques demeurées dans le bloc initial comme séquelle des anciennes identifications.

Le trou à boucher comporte maintenant seize pages. En vertu du principe du " plus court chemin ", il nous faut tenter d'y introduire les Capricci qui occupent actuellement cette place, à savoir le Cuchu et La sol fa re mi. Il y a à cela plusieurs raisons : nous avons observé au début que le volume se scindait nettement en deux (ou trois) blocs par la pagination et le foliotage. Il serait illogique de tenter de mélanger ces blocs dont la partition même paraît avoir pour origine les états que nous tentons de reconstituer. Les Capricci qui nous manquent doivent en toute vraisemblance être empruntés au bloc dont proviennent ceux que nous avons rétablis. D'autre part, les caractères utilisés pour les titres ont ici leur importance: les titres des six Capricci du premier bloc sont en gros caractères; il n'y a aucune raison de penser que le typographe qui, comme nous l'avons supposé, procède au fur et à mesure de l'arrivée des oeuvres, éprouve soudainement et sans fondement pratique le désir de changer de caractères pour revenir aux caractères initiaux dès la Fiamenga.

Nous allons cependant rencontrer un nouveau problème : 
l'espace vacant est de seize pages, et les Capricci en question en totalisent dix-sept (huit pour le Cuchu et neuf pour La sol fa re mi). Une page doit donc sauter, et c'est à nouveau une double barre inespérée qui nous tirera d'affaire. La deuxième page du Cuchu (page 24 actuelle) non seulement se termine en effet sur la fin d'une variation, avec une double barre, mais également s'ouvre sur le début d'une variation ternaire; il s'agit ainsi d'une page parfaitement autonome, qui présente la caractéristique supplémentaire de l'oubli du titre dans tous les exemplaires conservés. Tout se passe comme si cette page, typographiée après l'achèvement de la version en discussion et originellement destinée à clore le morceau, avait été subséquemment introduite à cet endroit. Les custodes qui terminent la page précédente ne contredisent nullement l'hypothèse : comportant de toutes façons deux erreurs, ils peuvent renvoyer aussi bien aux ténor et soprano de la page 24 que de la page 25. De cette page qui sera discutée plus loin, nous pouvons admettre pour l'instant, sans risque d'erreur, qu'elle constitute un transfuge et qu'elle ne faisait pas partie intégrante de la version originale. Le Cuchu se trouve ainsi réduit à sept pages, ce qui nous permet d'introduire sans problème les deux Capricci dans notre reconstitution.

L'ordre dans lequel ils doivent l'être est probablement inverse de leur ordre actuel. Il nous faudra en effet, à un moment ou à un autre, rendre compte des irrégularités dans l'identification par paires du bloc initial dans son aspect définitif (voir Tableau I). Le principe est de considérer ces lettres comme des séquelles d'identifications antérieures plutôt que comme un nouveau "foliotage » : la pagination d'une part, dans l'état définitif, suffit à sa correcte ordonnance ; d'autre part, il était probablement plus facile de substituer une nouvelle lettre dans l' "adresse " de foliotage à une ancienne périmée que de supprimer toute l'adresse. Il n'était en revanche pas nécessaire pour la mise en ordre finale de compléter les indications totalement absentes, puisque la pagination y pourvoyait. Il nous faut donc procéder de façon à faire concorder autant que possible, dans notre reconstitution, les pages identifiées avec des situations hypothétiques rendant cette identification nécessaire. C'est ce raisonnement même qui nous servira à reconstruire, plus loin, l'étape «intermédiaire " par laquelle se justifiera le trou de six pages du bloc central, et à l'analyse de laquelle nous renvoyons le lecteur en ce qui corcerne l'élucidation du cas présent.

Ainsi se trouve reconstituée cancrizans la fin du volume, du 
Capriccio Ut re mi fa sol la à la Spagnoletta. Tout comme le chant des Ungeborenen de la Frau ohne Schatten se fait urgent maintenant l'appel du début.

Avant la première page du Capriccio Ut re mi fa sol la et déduction faite des trois pages initiales de texte, nous disposons donc de trente-deux pages. Si nous additionnons les pages des Capricci restants à l'exception d'Or chè noi rimena, nous allons parvenir à trente-trois pages : à nouveau le puzzle recèle une pièce de trop, et il faut chercher la faute.

Il est exclu de remettre en cause les résultats acquis jusqu'ici : plus rien n'aurait de sens. Si l'on veut bien se rappeler que notre reconstitution se fait à rebours du sens suivi par le processus que nous reconstruisons, on sera mieux armé pour démasquer la page excédentaire.

Nous avions suggéré au début de notre enquête que le foliotage en minuscules attribué au bloc final représente un " nouveau départ " après le désordre qui précède, destiné à lever toute ambiguïté quant à la position des cahiers dans le volume, et nous en avions déduit que ce bloc était donc un transfuge. Il est de ce fait indubitable qu'il s'agissait bien du groupe de pièces que nous cherchons présentement. Que tel soit le cas, et c'est alors précisément ce groupe dont nous avions présumé plus haut qu'il constituait le premier ensemble "définitif ", avec les deux Capricci « napolitains» (Durezze et Cromatico), dont Frescobaldi pensait composer son volume, cela sur la base des caractères identiques employés pour les titres et de la planification des cahiers de trois folios faite par Soldi. Tout au long de cegroupe de cinq Capricci, la continuité dans la mise en page de la musique n'y autorise aucun prélèvement de page. Or comme le bouleversement a pris place une fois la ty pographie terminée, il est irréaliste d'y supposer des transformations musicales : nous devons donc nous en tenir au contenu tel qu'il s'y présente actuellement. La solution de l'énigme doit par conséquent être recherchée dans les pages de texte initiales.

Que la dédicace doive être remise en cause est peu vraisemblable : on ne voit pas pourquoi Frescobaldi aurait publié l'ouvrage - même beaucoup plus bref - sans l'appui financier qu'elle devait entraîner. Tout au plus pourrait-on penser que c'est après avoir obtenu l'assurance d'un financement par Alfonso d'Este que 
Frescobaldi s'est décidé à allonger le contenu en augmentant par là la dépense. ${ }^{12}$ La solution la plus plausible est que Frescobaldi n'ait conçu son avertissement au lecteur que plus tard, au vu de l'importance qu'avait prise entre-temps le volume et des difficultés d'exécution qui s'y accumulaient. Et ce serait ainsi précisément l'insertion tardive de cet avertissement qui aurait été partiellement responsable de la refonte que subira la publication. Si l'on considère ces cinq Capricci comme formant le noyau initial définitif, on s'apercevra en effet que ce sont ceux des Capricci qui auraient pu le plus aisément se passer des explications deFrescobaldi : on n'y rencontre guère plus d'une ou deux sections de style libre, et le tempo des triples y est plus facile à interpréter correctement. ${ }^{13}$ Notons au passage que ses Ricercari et Canzoni de 1615 pas plus que ses Fantaisies de 1608 n'avaient fait l'objet de commentaires en préface. Dans le cas des Capricci, Frescobaldi aurait donc agi un peu comme il l'avait fait pour la deuxième édition du premier livre des Toccate ou pour leur édition définitive - remaniant la préface au vu des transformations opérées dans la partition musicale. ${ }^{14}$

La question de la page excédentaire élucidée, le dernier problème à affronter sera à nouveau celui de l'ordre. L'argumentation des lettres-séquelles dans ce cas ne conduit à rien puisque le foliotage définitif de ce bloc est régulier et ne comporte pas d'adresse complète. On peut dire a priori qu'il serait fortement invraisemblable que Frescobaldi ait ouvert une nouvelle publication avec des compositions telles que les petits Capricci harmoniques (Durezze et Cromatico) ; parmi les grands Capricci, lequel sera donc le premier? Deux indices ici nous aideront : la Tavola donne celui avec Obligo di cantare la quinta parte (abrégé dorénavant «Quinta parte») avant celui sopra un Sogetto; plutôt étrange dans un volume constitué, une telle " erreur " s'expliquerait parfaitement sil'imprimeur avait simplement reproduit par inadvertance un ordre qu'il avait en mémoire depuis longtemps et auquel il était habitué. De supposer que la Quinta parte ouvrait le volume - et c'est une ouverture plutôt imposante-expliquerait en outre adéquatement l'usage des petits caractères de titres pour l'ensemble du bloc : pour un titre aussi long que Capriccio Obligo di cantare la Quinta parte, Senza toccarla, sempre di Obigo del Sogetto scritto, Si placet, il était é videmment impossible d'user de grands caractères ; par homogénéité, et en quelque sorte «mécaniquement ", l'imprimeur a continué avec les mêmes caractères pour les autres pièces de ces trois premiers cahiers. L'ordre initial 
serait donc celui qui termine la Tavola. Quant aux deux petits Capricci, il y a une raison sérieuse de penser qu'ils se présentaient dans l'ordre inverse de l'ordre actuel, et dont nous réservons l'explication pour la logique de l'exposé. Nous pouvons cependant d'emblée supposer que le Capriccio cromatico, plus imposant que l'autre, terminait mieux la première étape ou le premier « jet » du volume.

A ce point, il est possible de reconstituer un premier scénario vraisemblable. Frescobaldi amène à Soldi un groupe de cinq Capricci - Quinta parte, Sogetto, Ruggiero, Durezze, Cromatico - destinés ensemble à constituer un volume dont il ne peut évaluer exactement l'ampleur une fois imprimé. Cet ensemble, sans les avertissements agli studiosi, comprend un Capriccio énigmatique, un autre sur un sujet composé, un troisième sur une basse connue dont les segments serviront de sujets aux variations contrapuntiques, et deux Capricci " napolitains ", sur une recherche harmonique et sur un obligo contrapuntique. Cet ensemble forme en fait un beau condensé de ce que représentera la totalité des Capricci dans leur aspect définitif. Soldi planifie alors son imprimé et répartit le tout en trois cahiers, réservant la page 36 pour une éventuelle Tavola.

S'avisant peut-être alors de la minceur du résultat, Frescobaldi complète la série de deux ou peut-être trois nouveaux Capricci, tous sur des sujets de solmisation qu'il n'avait pas encore exploités (Ut re mi fa sol la dans la version réduite de quatre pages, La so fa mi re ut et La sol fa re mi). Soldi trouve alors le «dossier » et projette deux cahiers de deux folios au moins plus quelques feuilles; il distingue son nouveau foliotage du précédent par l'ajout du chiffre « $\mathrm{l}$ » aux lettres. Il passe aux gros caractères pour les titres - l'une des raisons pour lesquelles il est probant d'admettre que ces trois Capricci ne faisaient pas partie du projet initial. C'est alors que, sur sa lancée, Frescobaldi continue d'alimenter l'ouvrage avec le Cuchu (dans la version de sept pages), et plus tard avec le couple Spagnoletta - Fiamenga dans cet ordre, pensant vraisemblablement terminer avec ce dernier. Soldi, dans l'incertitude, a entre-temps «folioté » par paires, ce qui le laissait libre quant à la disposition en cahiers.

Mais alors survient, à la suite d'un mauvais calcul d'espace, l'interversion des deux derniers Capricci; d'autre part le sixième cahier de deux folios (S) - dans l'arrangement qui s'était imposé entre-temps - présente un vide de trois pages avant la Tavola, à moins que ce folio $\mathrm{S}$ ait été destiné à clore le volume comme folio 
isolé, rempli dès lors par les quatre dernières pages de la Spagnoletta et excluant par le fait même l'insertion d'une Tavola. Pour l'une ou l'autre de ces raisons, Frescobaldi décide alors de boucher le trou de trois pages en introduisant un ultime Capriccio sur l'Aria Or chè noi rimena.

Ce Capriccio particulier appelle ici quelques commentaires. Essentiellement différent des autres, il est conçu selon le modèle de la «partita » frescobaldienne dont chaque variation est séparée des autres et numérotée à part. Le modèle sera d'ailleurs repris trois ans plus tard par Frescobaldi dans le Capriccio Fra Jacopino sopra l'aria di Ruggiero du second livre des Toccate. Il n'est de ce fait guère surprenant que Fresocbaldi l'ait retiré dès 1626 des éditions ultérieures des Capricci, ce dont il importe de se souvenir dans le contexte de la présente investigation.

Tout, dans cette optique, donne à penser que ce " corps étranger " parmi les Capricci représente une "oeuvre de réserve" à laquelle Frescobaldi aurait recouru pour utiliser ses dernières pages disponibles. L'examen de l'imprimé, de son côté, paraît en étayer fortement l'hypothèse ; les deux premières variations occupent deux pages entières terminées par une double barre après la deuxième. La troisième variation se termine à la fin de la page suivante : dans ce cas, on a manifestement voulu introduire une double barre, qui se trouve serrée à la fin de la ligne dans un espace très restreint. Si ces trois variations sont identifiées nommément (Prima, Seconda, Terza parte), la quatrième ne l'est pas, alors que deux pages plus loin, la cinquième comporte l'identification latine (et non italienne comme les trois premières) : quinta pars. De plus ces deux dernières variations occupent chacune cinq lignes de musique réparties sur deux pages (une page est normalement couverte par trois lignes) : la deuxième page dans chaque cas comporte deux lignes seulement, suivies d'un motif décoratif destiné à remplir l'espace d'une troisième ligne absente ; toutefois, la fin de la quatrième variation ne présente pas de double barre.

On relève ainsi deux "blocs " dans ce Capriccio, qui pourraient bien correspondre à deux étapes de fabrication : les trois premières pages sont solidaires par la conséquence des désignations italiennes des variations (prima à terza parte) et par l'effort manifeste pour introduire une double barre malgré le manque d'espace à la fin de la troisième. Les deux dernières variations quant à elles ont pu être typographiées ultérieurement : d'abord on oublie d'indiquer «quarta parte» et on se trompe en marquant 
quinta pars la cinquième; d'autre part tout se passe comme si on avait voulu occuper un nombre discret de pages - en l'occurrence quatre - en laissant des espaces libres au pied de deux pages, d'une ligne chacun, plutôt qu'un large espace libre au pied de la dernière page. En fin de volume, un tel espace n'aurait nullement dérangé : il eût même été possible d'y ménager la tavola, dans l'espace qu'elle occupe actuellement, et de terminer ainsi le volume avec ces quatre pages supplémentaires formant l'ultime folio. Cette disposition donne à penser que ces quatre pages ont donc été conçues ultérieurement et arrangées de façon à être introduites quelque part dans le corps du volume (dont les pages environnantes étaient déjà définies) plutôt qu'à la fin, sans provoquer de "rupture » importante dans la disposition continue de la musique.

Le volume dans son premier état se serait ainsi achevé par un dernier cahier de deux folios comportant, dès $\mathrm{S}$, les quatre dernières pages de la Spagnoletta, les trois variations initiales de $\mathrm{Or}$ chè noi rimena (notons que la longueur du titre a forcé Soldi à revenir pour cette pièce aux petits caractères de titre), et la Tavola. Dans le Tableau II qui suit, nous donnons la reconstruction de cette étape initiale, en indiquant le début de chaque Capriccio par le numéro encerclé de sa pagination dans la version définitive (Tableau I) ; de plus, afin de rendre plus intelligible l'étape suivante, nous ferons précéder d'un astérisque (*) les pages qui comporteront une identification de folio dans la version définitive (telle par exemple la deuxième page du Capriccio La sol fa mi re ut, qui deviendra la page 15 signée $H$ dans le foliotage final, comme le montre le Tableau I plus haut).

Cet état constitue un volume bien organisé de quatre-vingtquatre pages et fort homogène : après les cinq Capricci que nous avons déjà commentés (trois grands et deux petits), on y trouve trois Capricci sur des thèmes de solmisation et trois autres développant des motifs du patrimoine commun, de dimensions comparables. Seules dérangent la position excentrique et la brièveté du dernier.

Que s'est-il passé alors? C'est évidemment en comparant le contenu et l'ordonnance actuels avec ce premier état qu'on trouvera une explication. Les changements principaux y seront l'ajout de l'avertissement, l'élargissement du Capriccio Or chè noi rimena de trois à cinq variations et son déplacement au centre du volume, l'ajout d'une page au Cuchu et de six pages à Ut remifa sol la. Quant aux indices relevés en début d'enquête et non encore 


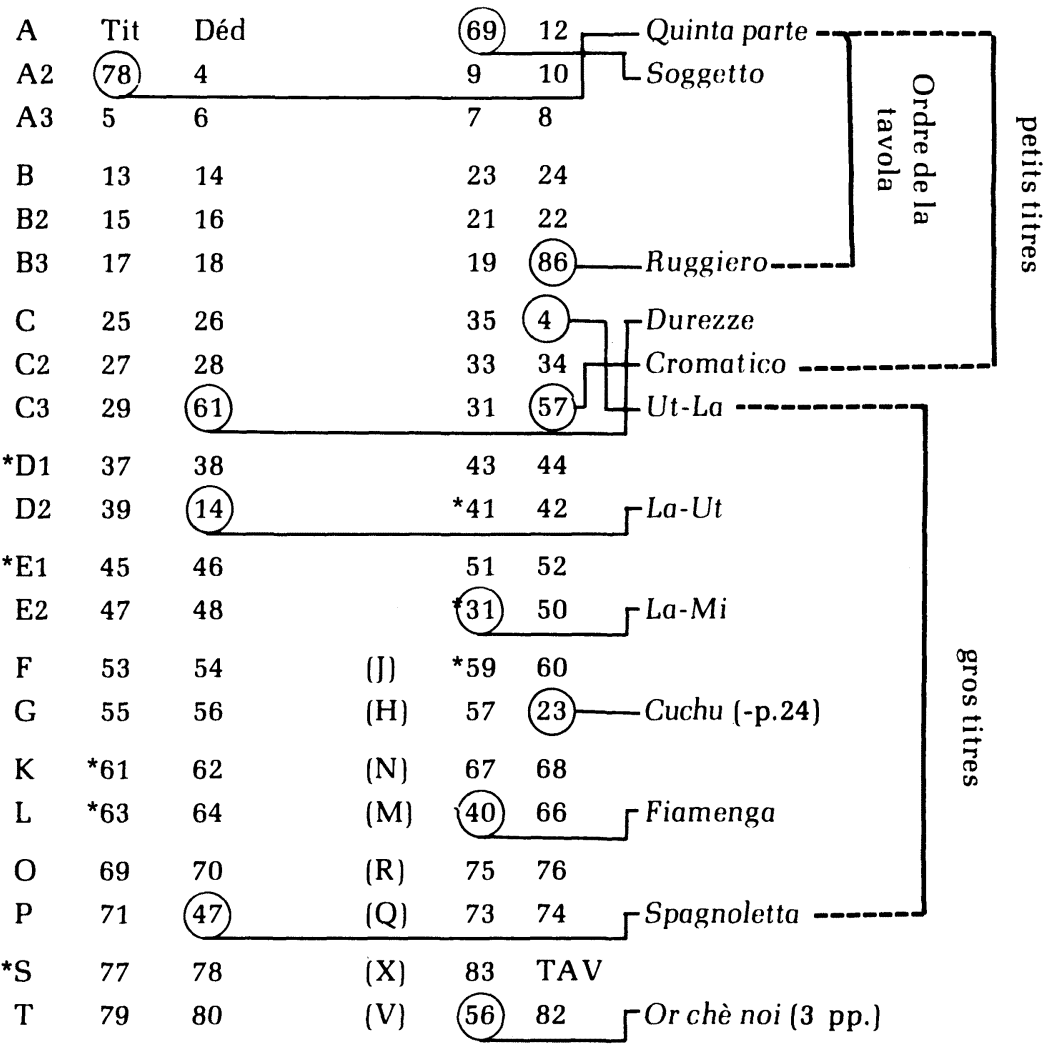

Tableau II

Reconstitution

Code : * = foliotages conservés dans la version définitive, ou pages qui en comporteront un avec "adresse".

(47)= origine du Capriccio dans la pagination de la version définitive. 
expliqués, l'erreur de pagination du bloc central est au premier plan, suivie des trous dans le foliotage initial par paires et de l'absence de pagination aux pages 57,68 et 69-95. Le moyen le plus simple pour rendre compte du processus est à nouveau d'imaginer un scénario où chacun de ces indices trouverait son explication. A ce point de l'enquête, l'éventail des possibilités est d'ailleurs passablement réduit, ce qui diminue d'autant le risque d'erreur. La difficulté principale sera de départager les changements fondamentaux - ceux qui ont motivé le bouleversement pour Frescobaldi - de ceux qui ont été réalisés simplement à l'occasion de ce remaniement.

\section{L'étape intermédiaire.}

Comme le révélera la suite de l'argumentation, il semble clair que le bouleversement qui eut lieu à ce point soit attribuable au problème du dernier Capriccio (Or chè noi rimena) ainsi qu'à la nécessité d'introduire une préface. Ce Capriccio trop bref et différent constitue une bien maigre conclusion pour un ouvrage de cette nouvelle ampleur. Frescobaldi décide alors de l'élargir de deux variations sans doute pour en assortir plus adéquatement la dimension à celle des autres; de plus il songe maintenant à introduire une préface d'explication à l'exécutant qui pourrait être découragé par l'apparente difficulté des compositions, surtout par celle des nouveaux grands Capricci ajoutés en fin de course, avec leurs nombreuses sections de styles et tempi contrastés. D'insérer une page au début signifie évidemment la refonte du volume avec son foliotage: il appert dès lors opportun de s'arranger pour équilibrer le volume de façon à "dissimuler " un peu le Capriccio dépareillé sur Or chè noi rimena (qui, rappelons-le, sera supprimé des rééditions) en le plaçant au centre, et en réservant ainsi la fin à un Capriccio plus brillant et plus représentatif. Afin de limiter au maximum les transformations dans la séquence des Capricci, la meilleure solution est d'ouvrir le volume avec le «bloc ajouté » du premier état - les Capricci Ut re mi fa sol la à Spagnoletta - dont les nombreuses identifications de foliotage pourront ainsi être partiellement réutilisées (cela d'autant plus qu'elle contenaient, outre les lettres, l' «adresse " complète de l'ouvrage); on insère ensuite Or chè noi rimena en typographiant l'ajout musical de façon à remplir au mieux un nombre discret de pages (quatre) puisque les pages qui suivront sont déjà faites et qu'il faut éviter de donner l'impression d'une rupture ; comme il n'est évidemment pas question de termi- 
ner le volume avec les deux petits Capricci napolitains (Cromatico et Durezze), on scinde par conséquent en deux le bloc initial de façon à terminer avec les trois grands Capricci du début : le Ruggiero, brillant et populaire, constituera un superbe couronnement à l'édifice.

A l'occasion de cette refonte, Frescobaldi revoit alors le Capriccio sopra il Cuchu auquel il ajoute une page, l'actuelle page 24 , dont tout cependant donne à croire qu'elle fut préalablement destinée à clore la pièce comme page finale : qu'on veuille bien se reporter ici à la discussion qui en fut esquissée plus haut. La présence d'une double barre en fin de page s'expliquerait mal autrement. D'autre partle motif développé au début de la nouvelle variation ternaire (Exemple a) apparaît plus directement dérivé du motif utilisé à la fin du Capriccio (Exemple b) que de celui avec lequel se termine la page initiale (Exemple c) et qui, maintenant, précède cette page 24 ; en revanche et à l'inverse, le nouveau motif de la page 25 (Exemple d) se rattache aisément au motif secondaire de la page 23 (Exemple e) alors qu'il est plus difficile de lui trouver un rapport avec la strette rapide en chutes de tierces ( «coucou ») qui termine la page 24, ce qui, dans notre démonstration, tient lieu de «contre-épreuve » convaincante :

Ex. a (mes. 24-26, alto)

Ex. b (mes. 142-43, ténor)

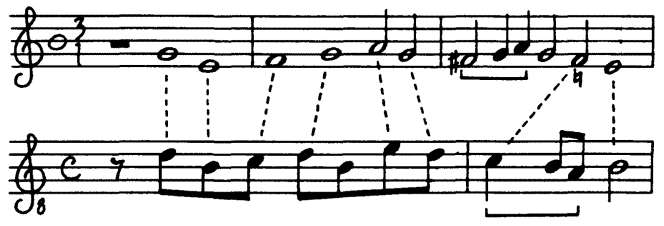

Ex. c (mes. 2-4, alto)

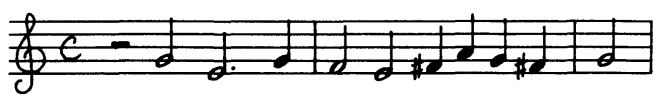

Ex. d (mes. 44, ténor)

Ex. e (mes. 6-7, alto)

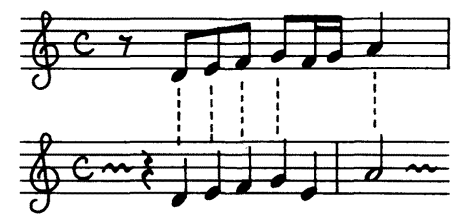

Enfin, nous avons montré dans notre préface à l'exécution ${ }^{15}$ que la succession d'une "mesure " (quantité entre deux barres) à quantité de brève, supposant un tactus alla semibreve alerte, et d'une mesure de "triple majeur " (tempus perfectum) à laquelle Frescobaldi, dans son avertissement, attribue le tactus le plus lent parmi les différents triples, présente un hiatus dans la 
logique du système par rapport au reste des Capricci : si nous admettons que cette page fut conçue pour clore le morceau - ce à quoi d'ailleurs sa dernière variation brillante se prêterait fort bien - cette difficulté s'évanouit puisque la notation diminuée en "mesures » de semibrèves quila précéderait suppose précisément un tactus nettement plus lent, permettant de ce fait une proportion idoine avec le triple majeur.

Si l'on s'est résigné en définitive à déplacer cette page déjà typographiée pour la ramener là où elle se trouve maintenant, en oubliant de supprimer la double barre mais en substituant toutefois des semibrèves aux brèves finales, c'est sûrement pour interrompre la monotonie métrique du début qui, sans cela, aurait présenté soixante-six mesures binaires avant le premier triple au lieu de vingt-trois. Dans son état actuel, la fin du Capriccio est moins brillante qu'elle ne l'aurait été dans le projet de modification initial (avec les alertes mesures 38 à 43), mais l'ensemble est plus varié et mieux équilibré du point de vue-essentiel pour les Capricci - des relations métrico-rythmiques.

L'ensemble des modifications discutées jusqu'ici représentent un total de six pages : l'avertissement, la page du Cuchu et les quatre pages d'Or chè noi rimena. Il est difficile de savoir si Frescobaldi pensait alors déjà à élargir le premier Capriccio (Ut re mi fa sol la) de quatre à douze pages ou si l'idée lui en est venue plus tard : il est évident en effet que Soldi, au cas où cet amendement à venir lui aurait été déjà connu, en oublia totalement l'existence comme va le démontrer l'argument suivant.

Si l'on écarte provisoirement l'échange des deux blocs extrêmes (Ut re mi fa sol la à Spagnoletta avec Quinta parte à Ruggiero), les principaux bouleversements d'ordre touchaient les Capricci Or chè noi rimena, Durezze et Cromatico. Afin de réorganiser son volume, Soldi calcule alors le nombre de pages du nouveau bloc initial modifié et arrive à quarante-neuf pages (quarante-cinq anciennes, plus une pour le Cuchu, plus trois pages de texte) : les plaques de ce bloc peuvent demeurer provisoirement intouchées car l'ordre demeure le même et il suffira d'en modifier le foliotage. Le même raisonnement vaut pour le bloc final en tant que bloc, son ordre interne n'important guère pour le moment à peu de choses près. En revanche il s'agit de définir et d'ordonner correctement les plaques du bloc central dont, rappelons-le, aucune ne comportait encore d'indication de foliotage : pour ce faire, et afin d'éviter toute erreur en l'absence de foliotage global, la solution la plus sûre est de les paginer en 
attribuant la page 50 au début de Or chè noi rimena, qui succédera aux quarante-neuf premières pages. Le fait que cette pagination (en petits caractères assortis au titre) se poursuit jusqu'à la page 61, la première page du Capriccio Durezze, indique que l'ordre initial de ces deux Capricci napolitains était effectivement inverse dans la version de départ (cf. le Tableau II) : si l'ordre en avait été le même que maintenant, il aurait en effet suffi, pour établir un ordre définitif, de paginer le seul Capriccio Or chè noi rimena, en considérant ces deux Capricci napolitains comme le pivot autour duquel devait être opérée la révolution des blocs extrêmes. Que cette pagination-repère partielle s'arrête à la première page du Capriccio Durezze le confirme : elle était suffisante pour indiquer univoquement que ce Capriccio devait suivre.

Ainsi se trouvent expliqués du même coup l'ordre que nous avions prêté à ces deux Capricci dans le Tableau II, le décalage de pagination dans le bloc central (Tableau I) et, évidemment, l'usage de petits chiffres pour cette première pagination: comme elle devait débuter avec Or chè noi rimena dont le titre était en petits caractères, le typographe s'est automatiquement servi de caractères identiques pour les chiffres.

Soldi alors réorganisa le début en cahiers de deux folios système dont il partait puisque le bloc concerné était formé de cette façon. Les nouveaux foliotages qu'il a dû commencer à introduire à cette occasion, modifiant d'ailleurs partiellement les anciens, le prouvent, qui expliqueront parfaitement les « résurgences » qu'on en observera dans le foliotage actuel en majuscules avec " adresse » de l'oeuvre. Le Tableau III qui suit reproduit ce nouveau résultat intermédiaire.

On l'aura remarqué, le tableau - en plus des nouveaux foliotages introduits dans cette version - indique à la fois les pages (soulignées) qui avaient fait l'objet d'un premier foliotage et donc comportaient l'adresse de l'oeuvre et celles ( ${ }^{*}$ ) qui en présenteront un dans la version définitive. En cumulant ces informations, on constatera que se trouvent ainsi justifiés tous les foliotages présents dans la version définitive, qu'ils proviennent soit de la version initiale que Soldi, à ce stade, n'avait sûrement pas pris la peine d'épurer, soit de la présente version intermédiaire ; la comparaison des Tableaux II et III le montre clairement : les pages 41, [49] et 59 du Tableau II, comportant un foliotage dans la version définitive, se trouvent dans la nouvelle colonne de gauche du Tableau III, comme pages 9, [25] et 19.

C'est guidé par ce même principle que nous sommes parvenu 


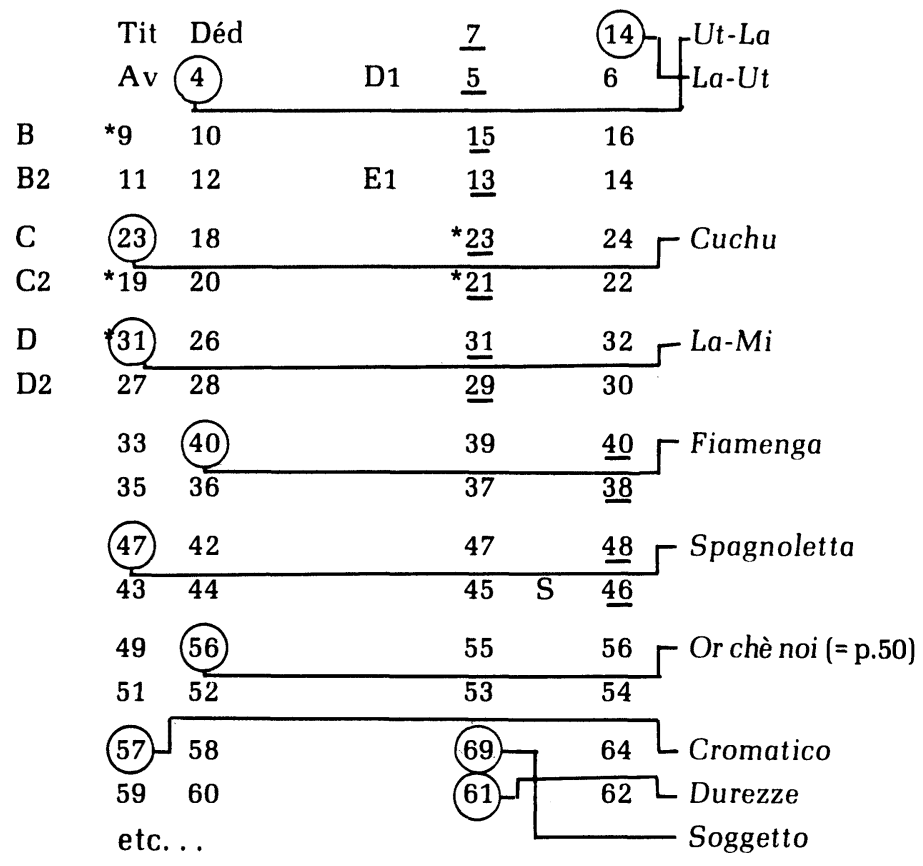

Tableau III

Étape intermédiaire

Note : pp. 52 à 61 = pagination erronée dans la version définitive.

Code : * pages qui comporteront un foliotage pertinent dans la version finale.

$\underline{38}$ (chiffres soulignés) = pages qui comportaient vraisemblablement un foliotage dans la première version. 
à la conclusion que les Capricci sopra il Cuchu et sopra La sol fa re mi avaient dû occuper l'ordre inverse dans la première étape (Tableau II) : on n'aura pas manqué de le remarquer en comparant les deux tableaux. Cet ordre initial était d'ailleurs plus logique d'un certaine façon, en ce qu'il regroupait les trois Capricci rendus solidaires par l'usage des motifs de solmisation. La raison de cette inversion d'ordre ne peut être qu'objet de conjectures : Frescobaldi a-t-il voulu plus de variété dans son premier bloc? Ou alors, songeant déjà à élargir le premier Capriccio, a-t-il voulu ainsi l'apparier au second dont le motif générateur constituait l'inversion de celui du premier (Ut re mi fa sol la versus La sol fa mi re ut)? Si cette dernière présomption s'avérait exacte, elle confirmerait que la mise en ordre de la deuxième étape (Tableau III) résulta, comme telle, d'un malentendu entre Frescobaldi et Soldi quant aux amendements à venir, malentendu bien compréhensible d'ailleurs dans le contexte des atermoiements du compositeur : Frescobaldi aurait annoncé à Soldi l'ajout de deux fois six pages et ce dernier aurait enregistré «six pages » seulement.

Afin de clarifier la question de l'ordre de ces deux Capricci, nous proposons ici une contre-épreuve où l'ordre, inchangé, aurait été dès la première étape celui de la version définitive :

\begin{tabular}{|c|c|c|c|c|c|c|c|}
\hline E1 & & & $\begin{array}{l}51 \\
23\end{array}$ & $\begin{array}{l}* 52 \\
* 50\end{array}$ & $\begin{array}{r}23 \\
* 19\end{array}$ & $\begin{array}{l}18 \\
20\end{array}$ & $\begin{array}{l}* 23 \\
{ }^{*} 21\end{array}$ \\
\hline$F$ & 53 & *54 & 59 & 60 & (31) & 26 & 31 \\
\hline 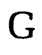 & 55 & *(31) & 57 & 58 & 27 & 28 & 29 \\
\hline & 61 & 62 & 67 & 68 & 33 & (40) & 39 \\
\hline I & 63 & 64 & (40) & 66 & 35 & 36 & 37 \\
\hline
\end{tabular}

On constate ici que les foliotages définitifs (marqués *) apparaissant aux pages 21 et 23 (version intermédiaire) ne trouvent pas d'explication dans la version initiale (pages 50 et 52), et que par conséquent, les lettres $\mathrm{O}$ et $\mathrm{P}$ du foliotage actuel (Tableau I) n'auraient été ajoutées que dans la dernière mise au point. Mais si tel avait été le cas, il serait difficile alors de comprendre pourquoi l'ensemble du foliotage définitif est si inconséquent et partiel, notamment dans la présence du " $Q$ » de la page 31. Comme nous l'avions pensé, il faut donc certainement comprendre ce foliotage ultime comme une " mise en ordre " hâtive et incomplète, aux moindres frais, d'états antérieurs dont les données ont été exploitées au maximum. 
Notre remarque suivante concernera précisément l'absence de foliotage après $Q$ dans la version finale - si l'on fait exception du S de la page 52. Celle-ci peut en fait s'expliquer facilement dans notre scénario : il faut noter ici que dès la page 40 (première de la Fiamenga), toutes les pages qui étaient impaires dans la version initiale deviennent paires dans la version intermédiaire, en raison de l'ajout d'une page au Cuchu (la comparaison des Tableaux II et III le montrera) ; en planifiant cette seconde version, Soldi pouvait donc dès cette page faire automatiquement sauter toutes les lettres et adresses de foliotage, car elles ne pourraient de toutes façons plus servir (par chance pour nous, il a oublié le "S " qui est à la clef de l'édifice). Il était obligé d'y refaire ex novo son foliotage. S'il ne l'a pas fait (comme le prouve l'absence de let tres dans la version finale), c'est que Frescobaldi a dû, précisément à ce point du travail, l'interrompre pour lui confier les six dernières pages du premier Capriccio - ou alors attirer son attention sur l'omission dont il s'était rendu coupable, au cas où Soldi aurait été en possession du manuscrit plus tôt. Pour la première partie du volume, tout le travail était alors à refaire.

\section{L'étape finale}

Tout at teste maintenant que les dernières étapes ont dû être conduites bien plus rapidement qu'il n'eût été souhaitable: si l'on tient compte des aventures encourues par le volume jusqu'ici, cette hâte est bien compréhensible au cas probable où l'on avait prévu un certain délai de publication. En possession des six pages à incorporer au Capriccio Ut re mi fa sol la, Soldi décide alors, à partir du cumul désordonné des deux foliotages antérieurs devenus caducs, de rafistoler tant bien que mal ce foliotage en fonction des cahiers qu'il prévoit, et pour lesquels il choisit de revenir à sa solution initiale de trois folios. Ce faisant, il commet quelques erreurs, oubliant de transformer D1 en C et de supprimer E1; il ajoute $G$ à la dernière des six nouvelles pages pour notifier l'ouverture du deuxième cahier, mais supprime par erreur le B2 de la version intermédiaire, qu'il aurait dû transformer en J. Après $Q$ qu'il a substitué automatiquement au $D$ antérieur bien que cela fût inutile, il arrête là ses efforts puisque, hormis une indication (supprimée) sur la septième page de La sol fa re mi et remontant au foliotage initial, les anciens foliotages avaient déjà été ôtés dans la version antérieure dès la page 40 (sauf $S$ ).

C'est alors qu'il se trouve confronté à l'ancienne pagination d'Or chè noi rimena qui s'avère évidemment décalée dans la 
nouvelle version : il corrige 50 en 56 , usant des mêmes petits caractères qu'il emploie à nouveau pour introduire 55. Arrivé à rebours à 54 où il commence à assortir les caractères à ceux des titres, il se trompe et place le chiffre à droite de la page. A ce moment il décide peut-être de reprendre toute la pagination dèsle début, ce qui lui garantira un ordre que le foliotage négligé et incomplet peut compromet tre. Il part de la première page numérotée (page 4), se trompe à nouveau de côté pour la page suivante, et termine la pagination de ce bloc.

Au moment de former le cinquième cahier (non folioté - cf. Tableau I), il décide, pressé en toute vraisemblance par le temps, de le réduire à deux folios de façon à constituer un sixième cahier autonome du bloc où demeure l'ancienne pagination erronée dont il renonce à poursuivre la correction après en avoir fait sauter le premier chiffre (51) : de réserver à ce sixième cahier un nouveau foliotage orthodoxe sera plus simple et plus rapide que d'en corriger la pagination, et suffisant pour en indiquer l'ordre d'impression des plaques. Comme il s'était arrêté à $Q$, assumant peut-être dès lors $R$ et $S$ pour les quatrième et cinquième cahiers, il choisit $\mathrm{t}$ minuscule $(\mathrm{t}-\mathrm{t} 2-\mathrm{t} 3$ ).

Pour terminer le volume, il lui restait alors sept folios qu'il a naturellement regroupés en trois cahiers de deux folios, plus un folio isolé, ce qui était clairement préférable pour la reliure, en leur attribuant un nouveau foliotage distinctif (a-b-c-d). S'il n'a pas poursuivi avec les lettres $v-x-y-z$, c'est qu'il devait passer à des cahiers de deux folios après le cahier $t:$ il distinguait ainsi les deux séries, ce qui lui évitait des erreurs d'impression ou de reliure. ${ }^{16}$ Il n'a cependant plus le temps de paginer ces ultimes cahiers.

Quant à la Tavola, elle avait étéévidemment composée juste a vant cette dernière mise au point du volume mais après l'ajout des six pages à $U t$ re mi fa sol la: elle reflète encore le décalage de pagination, mais relève le nombre exact de pages du premier Capriccio. Elle atteste enfin, comme nous l'avons mentionné plus haut, d'une nouvelle inversion d'ordre dans le dernier bloc (a-b-cd), où la Quinta parte passe de la première à la deuxième place. Comme elle donne une pagination qui ne figure pas entièrement dans le volume, elle a été du fait même réalisée séparément sur la base d'indications qui, tenu compte des bouleversements successifs, pouvaient difficilement n'en pas trahir quelques séquelles.

Un dernier aspect doit être encore brièvement évoqué ici : on trouve, dans les titres qui chapeautent chaque page, un certain 
nombre d'erreurs. Nombre d'entre elles ont été - parfois même inexactement - corrigées à l'aide de collettes après l'impression du volume. Que certains titres imprimés dans un exemplaire n'apparaissent pas dans un autre prouve qu'il y eut même des modifications en cours d'impression. Quant à l'absence pure et simple d'un titre - celui de la page 24 du Cuchu excepté - elle s'explique le plus adéquatement en admettant qu'erroné, le titre ait été enlevé de la plaque sans être remplacé.

On pourrait craindre que cet aspect remette en cause certaines de nos conclusions. Que les titres aient été réalisés au fur et à mesure de l'avance du travail - ou plus vraisemblablement à la fin d'une étape, ce qui expliquerait mieux certaines erreurs - ne changerait rien aux raisonnements que nous avons tenus, mais tendrait plutôt à les confirmer : ces méprises en effet consistent en échanges de titres entre les Capricci Ut re mi fa sol la et La sol fa mi re ut d'une part, et entre les trois derniers Capricci Sogetto, Quinta parte et Ruggiero d'autre part ; or nous avons précisément essayé de montrer que chacun de ces deux groupes avait fait l'objet d'u ne étape de réalisation séparée en ce qu'ils constituaient les " ensembles indépendants " que Frescobaldi fournissait à Soldi. Ces erreurs se comprennent dès lors facilement. Malgré la similitude de titres, il est en effet significatif qu'on ne trouve aucun échange entre La sol fa mi re ut et La sol fa re mi: si ce dernier Capriccio a bien été remis, comme nous le supposions, un peu après les précédents (dont les titres étaient faits), il eût été bien improbable que Soldi se fût trompé. En revanche, s'il n'a fait les titres des deux premiers qu'après en avoir terminéles plaques, les erreurs qu'ils recèlent s'expliquent fort bien. ${ }^{17}$

\section{Une esthétique " différente »}

L'ensemble de cette aventure, qui serait quasiment incroyable si la vraisemblance ne s'en trouvait étayée par la péripétie analogue que vivra, treize ans plus tard, le même Frescobaldi dans sa collaboration avec Nicolo Borbone, ${ }^{18}$ nous inspire quelques remarques de conclusion. Que Frescobaldi affirme dans sa préface que " celui à qui l'exécution de l'oeuvre entière causerait de la fatigue, pourra jouer jusqu'où bon lui semblera ces variations ( passi) et terminer sur celles qui finiront dans le ton $",{ }^{19}$ n'est plus guère étonnant : c'est la façon dont il les a apparemment 
pensées lui-même. Il ne concevait pas, comme l'a démontré notre enquête, ses oeuvres comme le " tout organique " auquel nous réfère notre héritage classique, puisque même au niveau de la composition, ces Capricci ont été ouvragés avant tout comme séquences de variations, certes plus intégrées qu'un ensemble tel que les Partite sur basse commune (la Romanesca par exemple), mais dont certaines sections pouvaient être omises sans dommage. Il n'est que de penser icià̀l'identique fragmentation qu'il autorisa, par ses avvertimenti al lettore de 1637 aux livres de Toccate, dans les partite sur Ciaccona ou Passacagli dont la séquence fut également le résultat d'une conception morcelée et amendée à moult reprises avant de trouver, dans l'imprimé final, son équilibre. ${ }^{20}$ Au dehors de la sphère même des imprimés frescobaldiens, on songe encore dans ce contexte aux surprenantes libertés prises par le copiste de la tablature de Turin, qui n'hésita pas à distribuer au fil des différents volumes de sa tablature les diverses sections d'un ensemble constitué, selon leur écriture ou leur genre: même d'une forme comme la Toccata vénitienne par exemple, les sections d'allure contrapuntique sont omises et parfois référées à d'autres parties de la tablature spécifiquement réservées à ce style. ${ }^{21}$

Un autre et dernier aspect digne d'être é voqué est celui de la conception du volume comme véritable «opus» : si l'ouvrage est le fruit d'ajouts successifs, il n'en demeure pas moins que les transformations qui sont intervenues dans l'ordre des pièces sans qu'un impératif pratique apparent ne le dictât, attestent l'importance qu'attribuait Frescobaldi à l'équilibre d'ensemble. Le souci prédominant y paraît être celui du contraste et de la variété des tempi et des styles d'écriture.

Quant à notre reconstitution de l'aventure, on pourra peutêtre en remettre en cause quelques détails. ${ }^{22}$ La rareté des indices de départ ne constitue toutefois nullement un argument pour en infirmer les conclusions : on aura compris qu'un ensemble de données qui, prises séparément, peuvent apparaître comme coïncidences ne sauraient plus être tenues pour telles dès le moment où, mises en relation les unes avec les autres, elles aboutissent à la formulation d'un écheveau d'hypothèses qui se confirment mutuellement pour expliquer un processus que, tenu compte des circonstances, le seul sens commun nous aurait suggéré. 


\section{NOTES}

1. Ce travail a été réalisé avec l'aide d'une subvention de recherche du Conseil de Recherche en Sciences Humaines du Canada, attribuée au projet intitulé : Edition critique des Capricci pour clavier de Girolamo Frescobaldi.

Notre édition - à laquelle se réfèrent les quelques numéros de mesure qu'on trouvera en cours de lecture - inclura : une partition critique faite à l'image des éditions originales de référence, une tablature destinée à faciliter la lecture, publiée en fascicule séparé et réalisée d'après le modèle des tablatures italiennes gravées et manuscrites de l'époque, un appareil philologique et critique, ainsi qu'une préface détaillée traitant des problèmes d'exécution (en particulier du temps). Les volumes - Girolamo Frescobaldi, Il Primo Libro di Capricci 1624 - paraîtront dans la série des Monumenti Musicali Italiani publiés par la Società italiana di musicologia, comme suite aux Opere complete de Frescobaldi, aux Edizioni Suvini Zerboni, Milan, en 1983.

2. Il primo libro di Capriccifatti sopra diversi soggettiet arie in partituradi Girolamo Frescobaldi organista in S. Pietro di Roma (armes des Este). In Roma. Appresso Luca Antonio Soldi MD.C.XX.IIII. Con licentia de superiori. Contenu : pp. [1-3], titre, dédicace, préface $A$ gli studiosi dell'opera ; puis 91 pages de musique et une Tavola. - Soldi, actif entre 1619 et 1625 , imprimait à S. Spirito in Sassia. Selon Boorman, il pourrait avoir été en relation avec Robletti ou Zanetti dont les imprimés lui sont revenus (cf. Boormann $1980: 449$ ).

3. Augmentés des Ricercari et Canzoni dont Bartolomeo Zanetti avait assuré l'édition originale à Rome en 1615, les Capricci ont été réédités trois fois, sans changement dans le titre et le contenu d'une édition à la suivante hormis les petites différences inhérentes à la réélaboration pour chacune de la typographie, in : Il primo libro di Capricci Canzon francese, e recercari ... in partitura, Venise, A. Vincenti 1626 ; id. 1628 ; id. 1642. Par rapport à l'édition princeps de 1624, ces rééditions n'offrent qu'une différence importante : la suppression du Capriccio opra l'Aria Or chè noi rimena.

4. Lors de la réédition de ses deux livres de Toccate en 1637, Frescobaldi a accru le premier d'une Aggiunta importante. Nous avons pu prouver que cet ajout fut initialement destiné au second livre et que son élaboration a fait l'objet de multiples transformations d'ordre et de contenu, dont les plus frappantes touchent à l'énigmatique série des Cento Partite sopra Passacagli. Le point crucial ici est que le processus, dont les étapes initiales peuvent être retracées dans les Manoscritti Chigiani de la Bibliothèque Vaticane (Chigi Q I 24, Q VIII 205-06 notamment), s'est poursuivi en cours de gravure de l'Aggiunta et non seulement au niveau de la préparation des manuscrits "définitifs". Cf. Darbellay [1983]: chap. II, publié comme appareil critique séparé à notre édition de ces livres : Girolamo Frescobaldi, Il Primo Libro di Toccate d'intavolatura di cimbalo e organo 1615-1637, a cura di É. Darbellay, Milano, Ed. Suvini Zerboni 1977 (Monumenti Musicali Italiani, vol. IV) ; Girolamo Frescobaldi, Il Secondo Libro di Toccate d'intavolatura di cimbalo e organo 1627-1637, ibid., 1979 (id. vol. V).

5. A titre indicatif, Vincenti foliotera son édition de 1626 (cf. note 3) en cahiers réguliers de quatre folios, et celles de 1628 et de 1642 , de papier très mince, en cahiers de huit folios.

6. Bergamo : Instituto G. Donizetti, Legato Piatti-Lochis, Cat. XIV ${ }^{\circ}$, no di cat. ${ }^{\circ} 8654$, Palchetto G1.

Berlin : Deutsche Staatsbibliothek Musikabteilung, Mus. ant. pract. F 755. 
Bologna : Biblioteca G.B. Martini, Z 170.

London: The British Library, 2 exemplaires : K.7.i.8 et Hirsch III 204.

Paris : Bibliothèque Nationale, Ancien Fonds du Conservatoire Rés. F 107.

Roma : Biblioteca Casanatense, Mus. 225.

Washington: Library of Congress, M7. F 88 C4 Case (Music 3091, item 2).

7. Les nombreuses petites différences que nous relevons dans l'appareil critique de notre édition ne sauraient consituer ici de critères utilisables. Il s'agit soit d'amendements manuscrits incomplets ou divergents soit de modifications intervenues en cours d'impression.

8. Dans la préface de son édition en partition des Canzoni instrumentales de 1628 de Frescobaldi, Bartolomeo Grassi attire l'attention sur le fait que le coût élévé de l'impression a empêché son maître de publier de nombreuses autres compositions demeurées ainsi manuscrites. D'autre part l'at titude de Frescobaldi lors des rééditions de ses deux livres de Toccate montre clairement qu'il mettait tout en oeuvre pour éviter au maximum de regraver une page déjà faite lorsqu'il voulait introduire une modification. Les seules exceptions connues sont les suppressions, dans la deuxième édition du premier livre de Toccate, de l'une ou l'autre variation ou fragment de variation, dans celle du deuxième livre, des deux séries finales de Ciaccone et Passacagli, et d'Or chè noi rimena dans les rééditions des Capricci. (Le cas particulier des Canzoni de 1628 ne peut être envisagé ici.) Cf. Darbellay [1983] : chap. Il. Enfin, dans le cas présent, il s'agit d'une première édition où tout indiquera, comme nous le verrons, que dut prédominer le processus inverse d'ajouts.

9. Comme on le verra, le contact étroit et permanent qui dut être maintenu entre le compositeur et l'imprimeur rend peu vraisemblable que Frescobaldi ait attendu l'achèvement du volume pour s'apercevoir de l' "erreur " du typographe. Ces quatre pages devaient réellement constituer le premier jet de l'oeuvre.

10. Si l'on admettait trois cahiers de deux folios ( 24 pages), on aboutirait à un total de soixante pages bien éloigné du total final de quatre-vingt-seize. Il s'agit de compenser le volume au plus près de son état définitif, de façon à retrouver par l'addition des pages antérieures et postérieures à D1 - E1 un total qui se rapproche le plus possible du nombre actuel. On verra que l'hypothèse de trente-six pages peut-être arbitraire à ce point - se vérifiera lorsqu'elle sera confrontée aux autres résultats de l'investigation.

11. Il s'agit de l'exemplaire coté K.7.i.8 de Londres : cf. note 6. La place correcte des liaisons et le $\mathrm{si}_{3}$ avec haste inversée sont indiqués dans le fac-similé. Les erreurs et ratures sont rapportées dans l'appareil critique de notre édition.

12. Cf. in note 8 la remarque de Grassi concernant les difficultés de financement des éditions. Il suffit en outre de rappelerici les tractations conduites en 1614-15 par Frescobaldi a vec le duc de Mantoue, dont il exigeait, pour abandonner Rome et entrer à son service, le financement convenu de l'édition du premier livre de Toccate (Darbellay [1983] : chap. II; Newcomb : 1977, 145-57).

13. La préface de notre édition (cf. note 1) tente d'élucider le rapport de tempo à observer entre les triples que Frescobaldi commente lui-même et les sections de mètre et rythme binaires qu'il n'évoque pas à cet égard mais dont la variété du Notenbild et des quantités métriques entre les barres constitue des critères décisifs quant à cette question. On trouvera également une discussion détaillée des problèmes de tempo soulevés par les séquelles de notation mensuraliste dans les livres de Toccate in Darbellay [1983] : chap. I.

14. Les avvertimenti al lettore des livres de Toccate et leurs variantes sont discutés in Darbellay [1983] : chap. I. 
15. Cf. note 1 et 13 ; il est vraiment significatif que, dans sa disposition actuelle, ce cas représente la seule incohérence à cet égard dans tout le volume, alors qu'en tant que fin de morceau, la notation de cette p. 24 serait parfaitement à l'unisson du " système ".

16. On peut s'interroger ici sur l'absence de toute séquelle de foliotage ancien dans le foliotage définitif de ce bloc. De fait, lorsque Soldi a déplacé vers le début le bloc qui occupe maintenant cette place, c'est évidemment de ce dernier qu'il a pris soin d'abord. Lorsqu'il a fallu revoir les foliotages du bloc final actuel, l'inversion des deux Capricci Quinta parte et Sogetto s'ajoutant à la nouvelle situation de cet ensemble, les confusions qui en auraient immanquablement résulté lui ont sûrement dicté la pure et simple suppression de tout foliotage résiduel. Quant au nouveau foliotage, il suffira à identifier a) l'ordre des deux Capricci, b) le passage au cahier de deux folios et c) la situation finale du bloc pris comme tel, c'est-à-dire se démarquant du précédent constitué d'un seul cahier de trois folios mais hybride.

17. Tous les détails de ces erreurs seront évidemment rapportés dans notre édition critique.

18. Cf. note 4 et Darbellay [1983] : chap. II.

19. ... che se l'Opere paressero di faticha il cominciar da principio fino al fine si potrà pigliar, dove piu piacerà di detti passi, et finire in quelli che termineranno del suo tuona ("A gli studiosi dell'opera », p. [3] ).

20. Li Passacagli si potranno separatamente sonare, conforme à chi piu piacerà, con agiustare il tempo dell'una e altra parte, cossi delle Ciaccone (On pourra jouer les passacailles séparément, au choix de l'exécutant, en prenant soin d'ajuster le tempo d'une variation avec l'autre ; il en est de même pour les chaconnes). Le point 2 du même avertissement attire également l'attention de l'exécutant sur la possibilité d'abréger les Toccate que le compositeur a pris soin de «fournir généreusement d'affetti et de passages variés».Cf. notre édition de ces volumes (voir note 4).

21. Il s'agit des seize volumes de la Raccolta R. Giordano, conservés à la Bibliothèque Nationale de Turin, rédigés en tablature d'orgue allemande. On y trouve notamment un important répertoire de musique italienne de clavier présentant nombre de concordances avec l'oeuvre imprimé de Fescobaldi.Cf. Darbellay [1983] : chap. III ; Mischiati 1963.

22. Depuis la soumission du présent article, l'explication qui concerne l'inversion de l'ordre des Capricci 5 et 6 (Spagnoletta, Fiamenga) a été légèrement modifiée. Dans sa forme nouvelle, elle rend compte plus clairement du projet initial et éclaire en outre la présence de doubles barres, dans Or che noi, non seulement après la troisième mais encore après la deuxième variation. Le lecteur intéressé pourra se référer à l'appareil critique conjoint des Toccate et Capricci à paraître en 1983. 


\section{RÉFÉRENCES}

BOORMAN, S.

1980 : "Soldi, Luca Antonio ", in The New Grove Dictionary of Music and Musicians. Stanley Sadie, éd. London : Macmillan, XVII, 449.

DARBELLAY, É

[1983]:Histoire, exécution et appareil philologico - critique des deux livres de Toccate de G. Frescobaldi. Milan : Suvini Zerboni (à paraître en italien).

MISCHIATI, O.

1963 : "L'intavolatura d'organo tedesca della Biblioteca Nazionale di Torino ", L'Organo, IV, 1-154.

NEWCOMB, A.

1977 : « Girolamo Frescobaldi, 1608-1615 : A Documentary Study », Annales musicologiques, VII, 111-58. 